\title{
Mapping cognitive and emotional networks in neurosurgical patients using resting-state functional magnetic resonance imaging
}

\author{
Michael P. Catalino, MD, MSc, ${ }^{1,2}$ Shun Yao, MD, PhD, ${ }^{1,3}$ Deborah L. Green, PhD, ${ }^{4}$ \\ Edward R. Laws Jr., MD, ${ }^{1}$ Alexandra J. Golby, MD, ${ }^{1}$ and Yanmei Tie, PhD, MSc ${ }^{1}$
}

Departments of ${ }^{1}$ Neurosurgery and ${ }^{4}$ Neurology, Brigham and Women's Hospital, Harvard Medical School, Boston, Massachusetts; 2Department of Neurosurgery, University of North Carolina Hospitals, Chapel Hill, North Carolina; and 'Department of Neurosurgery and Pituitary Tumor Center, The First Affiliated Hospital, Sun Yat-sen University, Guangzhou, China

\begin{abstract}
Neurosurgery has been at the forefront of a paradigm shift from a localizationist perspective to a network-based approach to brain mapping. Over the last 2 decades, we have seen dramatic improvements in the way we can image the human brain and noninvasively estimate the location of critical functional networks. In certain patients with brain tumors and epilepsy, intraoperative electrical stimulation has revealed direct links between these networks and their function. The focus of these techniques has rightfully been identification and preservation of so-called "eloquent" brain functions (i.e., motor and language), but there is building momentum for more extensive mapping of cognitive and emotional networks. In addition, there is growing interest in mapping these functions in patients with a broad range of neurosurgical diseases. Resting-state functional MRI ( $r s-f M R I)$ is a noninvasive imaging modality that is able to measure spontaneous low-frequency blood oxygen level-dependent signal fluctuations at rest to infer neuronal activity. Rs-fMRI may be able to map cognitive and emotional networks for individual patients. In this review, the authors give an overview of the rs-fMRI technique and associated cognitive and emotional resting-state networks, discuss the potential applications of rs-fMRI, and propose future directions for the mapping of cognition and emotion in neurosurgical patients.
\end{abstract}

https://thejns.org/doi/abs/10.3171/2019.11.FOCUS19773

KEYWORDS cognition; emotion; brain mapping; networks; resting state; fMRI; neuropsychology

$\mathrm{F}$ UNCTIONAL brain mapping was traditionally studied for epilepsy and tumor surgery by intraoperative direct electrical stimulation (DES) pioneered by Penfield, Ojemann, Berger, and Duffau. 2,14,43,46 Motor and language mapping has been well studied and broadly adopted, but cognition and emotion mapping is rare. ${ }^{28,60}$ In the last decade, our anatomical understanding of brain function has evolved from a purely localizationist perspective to a network-based approach. ${ }^{13}$ Indirect functional network mapping with resting-state functional MRI (rs-fMRI) has recently gained popularity as a way to noninvasively localize functional networks. ${ }^{33}$ Here, we review the methods for mapping cognitive and emotional networks using rs-fMRI and discuss possible applications in neurosurgical patients.

\section{Resting-State fMRI}

The emergence of blood oxygen level-dependent
(BOLD) fMRI makes it possible to noninvasively visualize inferred brain activity when the subject is performing a certain task. ${ }^{32}$ BOLD fMRI has entered clinical settings primarily for preoperative mapping of eloquent cortex in neurosurgical patients. ${ }^{44}$ Unlike the task-based fMRI that detects brain activation during performance of a certain task, rs-fMRI measures spontaneous low-frequency (< $0.1-\mathrm{Hz}$ ) BOLD signal fluctuations at rest. ${ }^{19}$ Rs-fMRI was introduced by Biswal et al. in $1995^{3}$ and has evolved as a technique to depict and quantify certain pathophysiological characteristics of human brain networks. ${ }^{50}$ The rsfMRI acquisition does not require that patients perform a task, which, unfortunately, makes inferring function more challenging. ${ }^{34}$ However, resting signal fluctuations have been linked to both structural anatomy of human white matter pathways and neuropsychological outcomes, which increases the confidence in the functional significance of these network measurements. ${ }^{66}$

ABBREVIATIONS BOLD = blood oxygen level-dependent; DAN = dorsal attention network; $\mathrm{DES}=$ direct electrical stimulation; $\mathrm{DMN}=$ default mode network; EEG = electroencephalography; FC = functional connectivity; fMRI = functional MRI; FPN = frontoparietal network; ICA = independent component analysis; ROI = region of interest; rs-fMRI = resting-state fMRI; RSN = resting-state network; SCA = seed-based correlation analysis; SN = salience network; VAN = ventral attention network

SUBMITTED September 30, 2019. ACCEPTED November 13, 2019.

INCLUDE WHEN CITING DOI: 10.3171/2019.11.FOCUS19773. 


\section{Rs-fMRI Analytical Methods}

Rs-fMRI analytical methods include voxel-based and node-based approaches. Among the voxel-based approaches, the seed-based correlation analysis (SCA) and independent component analysis (ICA) are most commonly applied. Other approaches, such as the amplitude of low-frequency fluctuations ${ }^{73}$ and regional homogeneity, ${ }^{72}$ have been employed to characterize local signal. Surgical treatment of brain lesions will invariably involve not only regional effects (e.g., local tissue damage) but also global network effects. ${ }^{25}$ Thus, if focal lesions can have global network effects, ${ }^{18}$ a comprehensive approach to brain network mapping may enhance the planning and delivery of surgical intervention.

\section{Seed-Based Correlation Analysis}

SCA was the first method to reveal brain functional connectivity (FC), and it has become a popular method to identify resting-state networks (RSNs). ${ }^{3}$ SCA can describe the FC of all brain voxels with respect to a defined region of interest (ROI). The main advantage of this hypothesisdriven SCA approach is the ability to address a specific question that relates to the FC of an ROI. However, it can be challenging to accurately define the seed region, especially if the ROI is structurally distorted.

\section{Independent Component Analysis}

Compared with the SCA, ICA applied to rs-fMRI data is a fully data-driven approach that decomposes the BOLD signal into a set of spatially independent components without prior assumptions. ${ }^{38}$ ICA can be applied to both task-based and rs-fMRI data and has been widely adopted in the identification of the motor and language networks for preoperative functional mapping. ${ }^{53,65,74}$ It is also attractive for rapid clinical application, as there is no need to manually define a seed region. However, selection and interpretation of the components can be challenging, ${ }^{24}$ and ICA parameter settings can influence the resulting networks (e.g., setting a large number of components may lead to potentially split networks). ${ }^{9}$

\section{Node-Based Connectivity Analysis}

The node-based connectivity approaches include network modeling, graph theory, dynamic causal modeling, and dynamic measures,${ }^{17}$ which have been widely used to explore the property and strength of FC of complex network systems. ${ }^{61}$ The most critical step of node-based connectivity analysis is to define the nodes based on brain parcellation or ROIs and edges (i.e., the strength of connectivity between each pair of ROIs). ${ }^{16}$ The result of this approach is a connectivity matrix or graph, instead of a brain map; therefore, it is more applicable to describe functional connections among a specific set of regions that together form a cognitive system.

\section{Identifying Eloquent Cortex}

The most common application of rs-fMRI in preoperative mapping is to identify traditionally eloquent cor- tex, i.e., mainly the regions involving motor control and language processing. In its early development, Tie et al. proposed and studied the feasibility of mapping language areas based on rs-fMRI data using a semiautomated approach for the identification of language components from ICA output. ${ }^{64}$ Later, Lu et al. proposed an automatic algorithm of component identification to match language networks and achieved a level of sensitivity that was superior to the SCA approach. ${ }^{36}$ In 2018, Zacà et al. developed a promising toolbox, ReStNeuMap, to automatically extract the RSNs in neurosurgical patients. ${ }^{71}$ This toolbox provides the ICA results of different numbers of components and automatically identifies the motor, primary visual, and language networks. Their initial experience in 6 brain tumor patients indicated good spatial agreement among the eloquent networks using rs-fMRI and DES awake mapping.

\section{Identifying Cognitive and Emotional Networks}

Neurosurgery, possibly more than most other surgical specialties, involves balancing the risks and benefits of treatment to achieve an optimal functional outcome. Besides preservation of traditionally eloquent function, neurosurgery is increasingly focused on preserving other less discrete functions (i.e., those associated with cognitive and emotional networks that can be mapped using rs-fMRI). These networks are more difficult to identify than motor, sensory, and even language networks. ${ }^{47}$ In our experience, it is uncommon to discuss neuropsychological outcomes with patients with focal lesions partly because of lack of confidence in the potential outcomes. To start, there are 5 groups of RSNs one might consider in this discussion, i.e., default mode, frontoparietal, salience, attention (dorsal and ventral), memory and emotional networks, and they can be categorized based on the brain functions they involve, i.e., self-awareness, arousal/attention, executive function, social behavior, memory and emotion..$^{55}$ Figure 1 shows an example of how SCA can identify 4 of the 5 presented networks that were acquired from rs-fMRI data obtained in a single patient with a right frontal glioblastoma. These RSNs can be revealed by both the SCA and ICA approaches. It is important to note that these networks are rarely considered "eloquent," and thus the longstanding effects of surgery would not be readily detected without comprehensive perioperative neuropsychological evaluations.

\section{Default Mode Network}

The default mode network (DMN) was initially discovered by meta-analysis of PET activation studies of the human brain..$^{57}$ Comparing the blood flow decrease across a wide range of tasks, Shulman et al. ${ }^{57}$ and Raichle ${ }^{50} \mathrm{ob}-$ served that the brain areas with decreased blood flow were largely task independent, suggesting a baseline mode of brain function. The DMN mainly consists of the posterior cingulate cortex, precuneus, lateral parietal area, medial prefrontal cortex, and medial temporal area. ${ }^{7}$ The DMN has been shown to be involved in multiple functions, including episodic memory, prospection, social cognition, and emotion. ${ }^{7}$ Rs-fMRI has shown FC between medial 


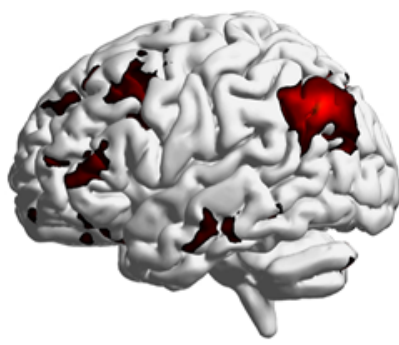

L
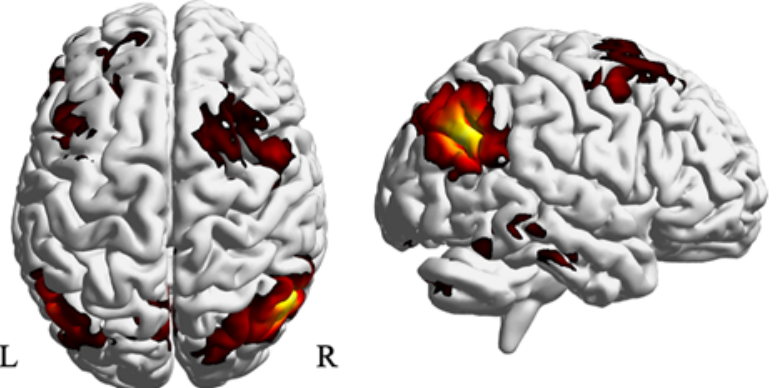

A. Default Mode Network

\section{1}

18.3

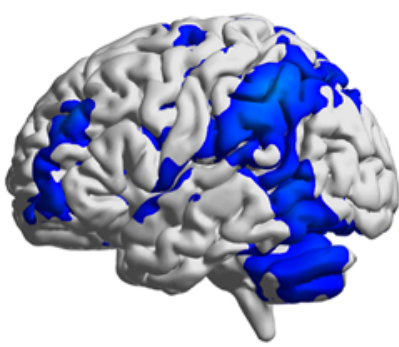

L
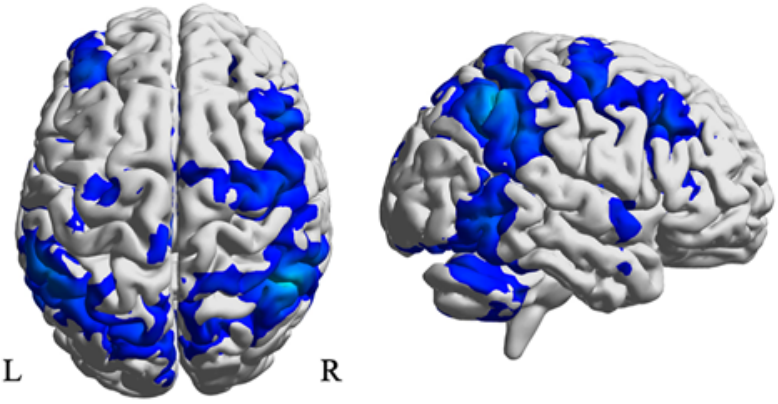

B. Frontoparietal Network

3.1

19.4
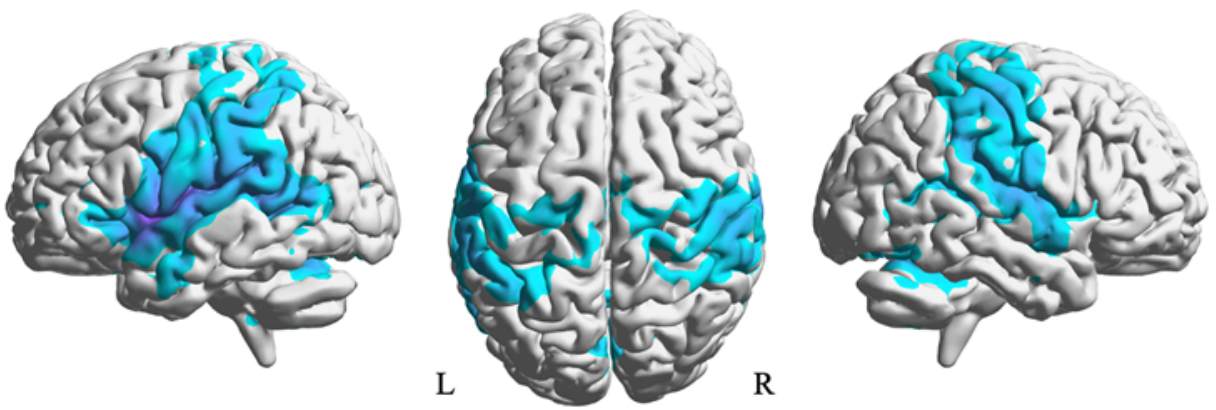

C. Salience Network

3.1

19.8

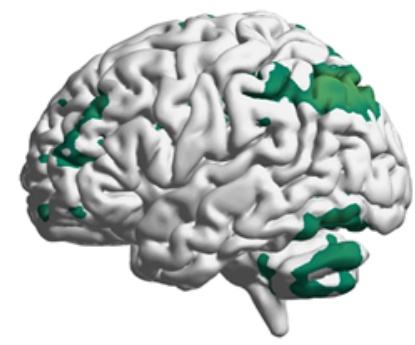

L
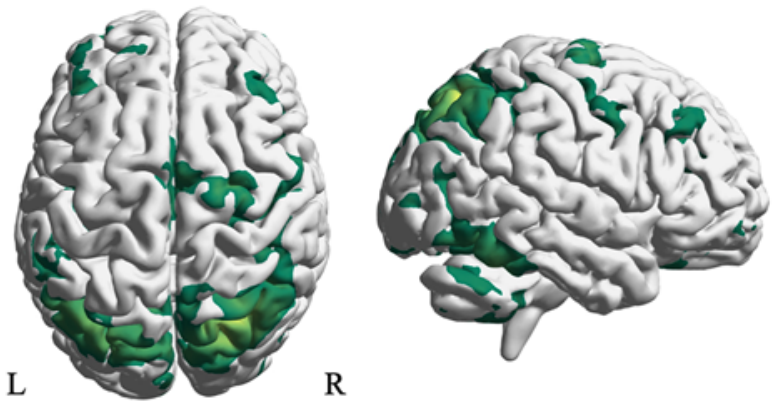

D. Dorsal Attention Network

3.1

19.9

FIG. 1. Three-dimensional views of the cognitive and emotional networks in a patient with a right frontal glioblastoma. All four RSNs were created by the SCA, using FEAT (FMRI Expert Analysis Tool) (version 6.00, part of FSL [FMRIB's Software Library v6.0, www.fmrib.ox.ac.uk/fsl]). Rs-fMRI preprocessing included head motion correction using Motion Correction FLIRT (MCFLIRT), nonbrain tissue removal using Brain Extraction Tool (BET), and registration with a postcontrast T1-weighted image that was normalized to the Montreal Neurological Institute (MNI) space. The seed regions were generated using an $8-\mathrm{mm}$ radius sphere centered on right lateral parietal (MNI coordinates: 49, $-63,33$ ), right superior parietal $(50,-51,45)$, left insula cortex $(-41,3,6)$, and right intraparietal sulcus $(26,-66,48)$ according to Raichle ME: The restless brain. Brain Connect $1: 3-12,2011$. Three of the four seeds were selected in the right hemisphere to demonstrate the networks that most likely correlated with the perilesional areas. For the SN, the left insular cortex was selected due to edema from the lesion infiltrating the right insula. The seed regions defined in the MNI space were then transformed to the patient's native space, and the average time series within the seed regions were extracted for correlation analysis with every voxel in the whole brain. The resulting map was then normalized to the MNI space for illustration. 
temporal areas of the DMN and frontal regions of the dorsal attention network (DAN), which are related to episodic memory; specifically, inversely correlated activity between these areas is associated with better verbal memory performance on the Hopkins Verbal Learning Test (total learning and delayed recall). ${ }^{63}$ Diminished FC within the DMN (between the posterior cingulate and medial prefrontal cortex) is associated with poorer performance on a computerized Stroop task, ${ }^{12}$ but the Trail Making Test A and $\mathrm{B}$, digit symbol test, and word fluency test were not correlated with DMN connectivity. Finally, hyperconnectivity of the DMN and frontoparietal network (FPN) has been implicated in major depressive disorder. ${ }^{30}$

\section{Frontoparietal Network}

The prefrontal and parietal areas show synchronous neural activity during diverse cognitive demands, suggesting that they may belong to a unified system, which is referred to as the FPN..$^{15}$ The FPN plays a critical role in the fast and flexible top-down goal-directed processes, namely executive control. ${ }^{10}$ Additionally, Dixon et al. identified two distinct subsystems within the FPN that have interactions with other RSNs, such as the DMN and the DAN. ${ }^{11}$ Kaiser et al. showed that major depressive disorder was associated with decreased FC of the FPN as well as hypoconnectivity of the FPN and parietal regions of the DAN responsible for attention to external stimuli. ${ }^{30}$ The FPN has shown high variability in its functional topography among individuals, calling for large-scale studies of its clinical usefulness. ${ }^{37}$

\section{Salience Network}

The salience network ( $\mathrm{SN}$ ) is a collection of interconnected large-scale networks wherein the anterior insula and dorsal anterior cingulate cortex are the most important hubs with three key subcortical structures: the amygdala, the ventral striatum, and the substantia nigra/ventral tegmental area. ${ }^{40}$ Intrinsically connected with other RSNs, the SN plays a key role in a variety of functions, including self-awareness and social behavior, by integrating sensory information, emotional processing, and cognitive functioning ${ }^{40}$ Additionally, the SN plays a role in keeping attention focused on the task-relevant goals by switching between the DMN and the FPN. ${ }^{39}$ Duchek et al. showed that diminished FC within the SN (between anterior cingulate and caudate) is associated with poorer performance on the computerized Stroop task, while SN-DMN connectivity was inversely correlated with Stroop performance. ${ }^{12}$

\section{Attention Network}

The attention network includes two separate networks: dorsal and ventral. ${ }^{68}$ The DAN consists of the gyri adjacent to the intraparietal sulcus and frontal eye field in both hemispheres, and it is active during the tasks involving voluntary attention. The DAN shows inverse correlation with the DMN. ${ }^{20}$ The ventral attention network (VAN) mainly consists of the temporoparietal junction and the ventral frontal cortex and is active when the external stimuli occur unexpectedly. The two attention networks are spatially isolated but jointly facilitate the response to top-down goals and bottom-up sensory stimuli. ${ }^{68}$ Zhang et al. studied the DAN in patients with mesial temporal lobe epilepsy who suffered from attention deficits. ${ }^{75}$ Although temporal lobe structures are typically associated with the VAN, the DAN has been well defined using rs-fMRI. The authors showed that patients with mesial temporal lobe epilepsy had decreased FC of the DAN compared with controls. Interestingly, performance on the Trail Making Test, a test of visual attention and task switching, was inversely correlated with the DAN FC z-score (increased or decreased FC), although others have not shown this same correlation in cognitively normal adults. ${ }^{12}$

\section{Memory and Emotional Networks}

The widespread memory networks are subdivided into three subsystems. The first system is the parietal memory network comprising the superior portion of the parietooccipital fissure, the portion of the cingulate cortex adjacent to the splenium, the posterior body of the corpus callosum, and the intraparietal sulcus. ${ }^{22}$ The parietal memory network mainly involves recognition memory functions. The second system is the medial temporal lobe memory network, which includes the hippocampal formation, the parahippocampal regions, and the entorhinal cortex. ${ }^{62}$ This system is essential to long-term encoding, storage, and retrieval of episodic or declarative memories. The third system is the parietooccipital network that consists of the parahippocampal cortex, the retrosplenial cortex, the superior portion of the precuneus, and the posterior part of the angular gyrus. This network mostly involves vision-related memory processing. ${ }^{1}$

The circuit of Papez was originally identified as an emotional network ${ }^{45}$ and this "limbic" network includes numerous cortical areas (orbitofrontal cortex, entorhinal cortex, hippocampus, and fornix), subcortical areas (septal nuclei, amygdala, and nucleus accumbens), and diencephalic structures (hypothalamus, mammillary bodies, and anterior nuclei of thalamus). ${ }^{48}$ Luiz Pessoa proposed a new emotional network model that emphasizes the importance of the large-scale network interactions in emotional processing across the entire brain, leading to the novel concept of "functional integration system" and "corticosubcortical systems." ${ }^{\prime 77}$ Studies have found that stress correlates with increases in the limbic/paralimbic amplitude of low-frequency fluctuations, whereas major depression may correlate with decreased signal. Similarly, anxious states showed increased FC within the subgenual anterior cingulate and ventral striatum, whereas depression inversely correlated with FC. ${ }^{42}$

\section{Rs-fMRI Mapping in Neurosurgery}

Although, at present, rs-fMRI is rarely used in the clinical setting, there are a few pragmatic advantages that make it attractive for neurosurgeons. 1) It is noninvasive and does not require specialized stimulus display equipment or a psychologist to administer the task assessment. 2) Network connectivity can be quantified at multiple time points for longitudinal follow-up. 3) It can be performed in children or in patients with compromised neurocogni- 
tive function. 4) It can assist with functional mapping in diseases not otherwise requiring awake surgery, such as neuroendocrine diseases and hydrocephalus. Leuthardt et al..$^{35}$ and Roland et al..$^{51}$ are pioneering the integration of rsfMRI into clinical practice using well-established imaging data pipelines. These centers have been able to integrate rs-fMRI into clinical practice by converting group mapping averages to individual patient-specific brain maps by training a multilayer machine learning algorithm to assign RSNs based on individual patient data. ${ }^{41}$

The nature of the neurological disease will also dictate to what degree rs-fMRI can be integrated into patient care. For example, in epilepsy and tumor surgery, rs-fMRI may have the potential to identify epileptic foci and map critical brain networks in order to assist the surgeon in performing more effective and safer resection of a brain lesion. The utility of rs-fMRI in these cases will depend on correlation studies that combine pre- and postoperative rs-fMRI, intraoperative DES, and stringent measurement of neuropsychological outcomes. For other diseases, like Cushing's disease and normal pressure hydrocephalus, where cognitive networks are affected but intraoperative DES is not possible, rs-fMRI may provide a more objective assessment of neuropsychological response to treatment if restoration of normal networks can be seen after surgical intervention.

\section{Epilepsy and Tumor Surgery}

The most likely initial avenue for neurosurgical application of rs-fMRI is for decision-making in epilepsy and tumor surgery. $4,5,33,35,41,51,56$ As an example, Boerwinkle et al. conducted a prospective study of pediatric patients with epilepsy and identified an rs-fMRI epileptogenic zone. ${ }^{4}$ This zone contained areas of deranged connectivity with atypical spatial patterns compared with norms used for noise, irregular time courses compared with those typical of established RSNs, and higher frequency $(>0.4 \mathrm{~Hz})$. Activation of these areas did not necessarily correlate with seizure activity, but the overlap of rs-fMRI epileptogenic zone and seizure onset zone on electroencephalography (EEG) correlated with seizure freedom after the operation. Seventy-five percent of patients with an unresected rs-fMRI epileptogenic zone continued to have seizures at 1 year. Among patients with concordance of rs-fMRI epileptogenic zone and EEG seizure onset zone, only $24 \%$ continued to have seizures at 1 year. Yordanova et al. studied 23 patients who underwent combined DES and rs-fM$\mathrm{RI}$ for resection of right-sided diffuse low-grade gliomas. ${ }^{70}$ Positive stimulation sites were used as seeds to map the network, formerly identified on task-based fMRI, involved in interpreting the emotional states of others. Thus, the authors were able to directly correlate function with discrete brain regions located within large-scale networks seen on imaging. These smaller-scale correlative studies complement the larger definitive descriptions of functional brain networks from probability maps based on DES results mapped to normalized brain space. ${ }^{27,54}$ The major limitation of these studies is that they required invasive DES for data acquisition.

\section{Cushing's Disease}

One emerging application of rs-fMRI is in investigating neuroendocrine-related neuropsychological outcomes. In healthy adults, learning and memory formation under stress has been linked to glucocorticoids and brain-derived neurotrophic factor inducing neural plasticity. ${ }^{23} \mathrm{In}$ addition to the known systemic morbidity from failure of circadian hypothalamic-pituitary-adrenal axis rhythmicity, patients with Cushing's disease experience cognitive, emotional, and memory problems hypothesized to originate from functional alterations in brain connectivity. ${ }^{52}$ Hippocampal atrophy often occurs in these patients..$^{59}$ Jiang et al. showed alterations in the rs-fMRI-derived amplitude of low-frequency fluctuations and regional homogeneity measurements in Cushing's disease patients compared with controls..$^{29}$ Other studies support a correlation between hypercortisolism and spontaneous activity of the DMN, which may support inefficient use of the DMN, similar to what has been shown in patients with psychiatric disorders and persistent hypercortisolism. ${ }^{49,67}$ Validation of rs-fMRI findings using gold-standard neuropsychological testing is needed, but this serves as an example of the types of pathology one can explore with the proper use of rs-fMRI methodology for studying cognitive and emotional networks.

\section{Idiopathic Normal Pressure Hydrocephalus}

Idiopathic normal pressure hydrocephalus is frequently diagnosed late in its course or misdiagnosed entirely, and patient selection for neurosurgical intervention is often an invasive, arduous, and ineffective process. ${ }^{69}$ The cognitive deficits in these patients involve prefrontal and subcortical regions, affecting extensive neurocognitive functions, such as memory, learning, attention, and executive function, as well as visuospatial processing. ${ }^{8,26}$ The characteristics of abnormal neural networks underlying these symptoms have not been extensively studied. One rs-fMRI study showed a reduced DMN connectivity compared with healthy controls, but neither other neural networks nor interactions among the networks were studied. ${ }^{31}$

\section{Rs-fMRI and DES Dissociation}

Rs-fMRI may be able to map beyond the traditionally eloquent areas assessed during DES. This "dissociation," or margin, might represent the less discrete functional network area beyond what is mapped during DES. Rs-fMRI has been shown to identify functional network locations with a low false-negative rate through such a dissociation between a novel data-driven RSN identification and DES. ${ }^{41}$ Boerwinkle et al. also identified this dissociation for seizure foci. ${ }^{4}$ These covert networks identified by rs-fMRI may not show functional manifestations in a clinically obvious way. With the shift from an "overt" or "eloquent" functional preservation paradigm to one more inclusive of neuropsychological functions, the rs-fMRIDES dissociation needs to be further studied in order to validate the clinical utility of overlapping and nonoverlapping rs-fMRI-DES areas. 
Seeds
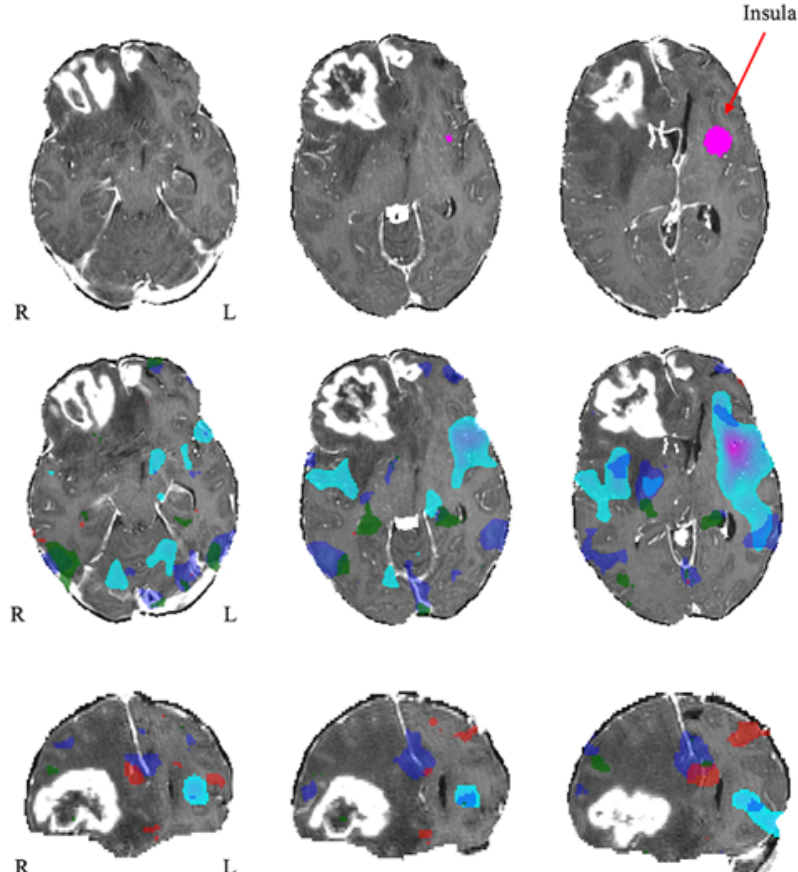

$\mathrm{L}$
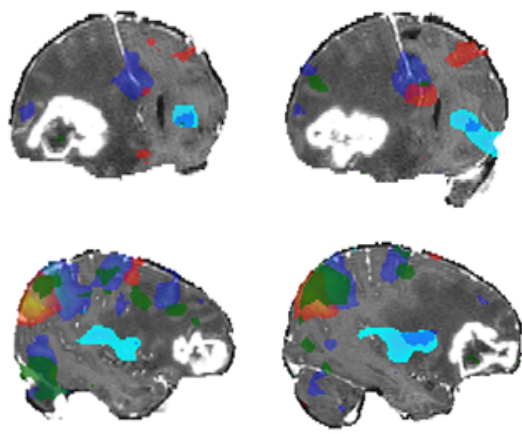

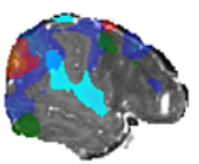

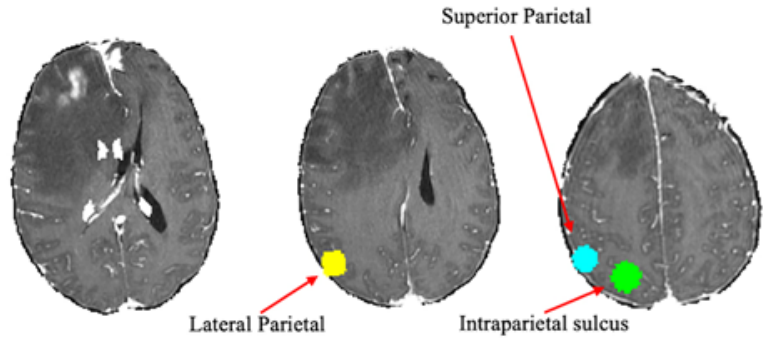
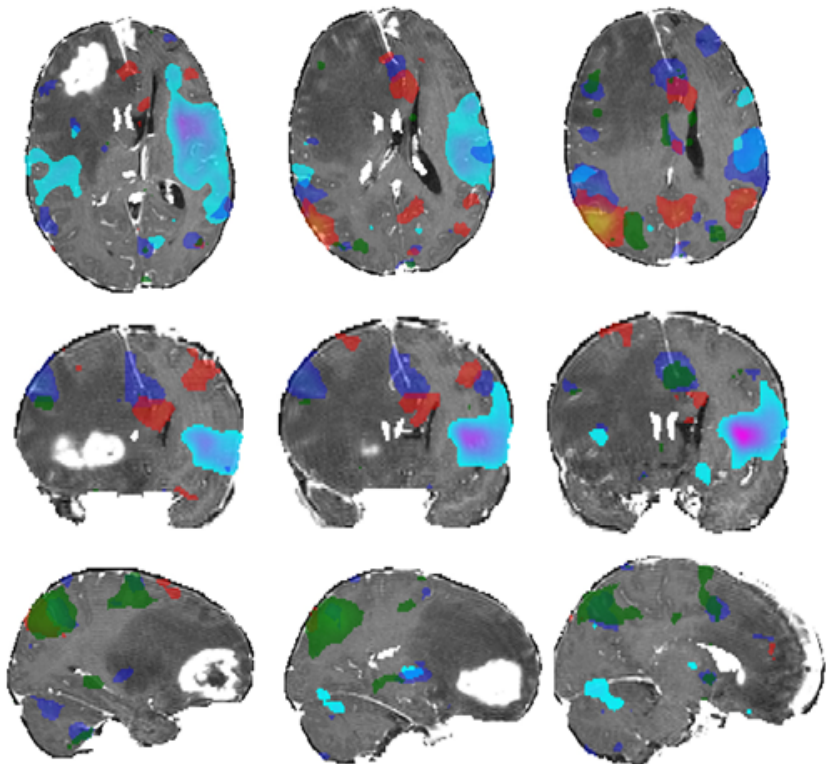

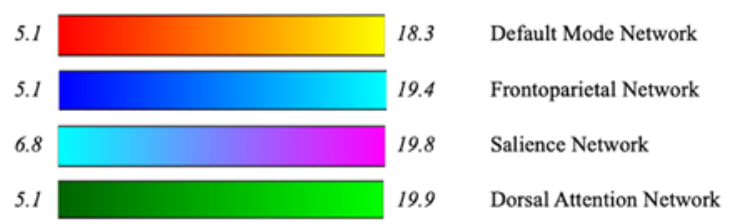

FIG. 2. Orthogonal views of the cognitive and emotional networks in a patient with a right frontal glioblastoma (the same patient as Fig. 1), overlapped onto the patient's postcontrast T1-weighted image. The RSNs were created by the SCA as described in Fig. 1. The seed regions are also shown.

\section{Limitations}

The main limitation of rs-fMRI remains the lack of direct measurement of neuronal activity, and, thus, function is inferred and not directly measured (as it is with DES). Therefore, before rs-fMRI can be appropriately deployed as a tool for mapping brain network function prior to surgery or evaluating treatment outcomes, it must be validated by rigorous, disease-specific, hypothesis-driven studies using gold-standard DES and/or neuropsychological assessment of outcomes. Additionally, there are several technical challenges and issues of variability of rs-fMRI that need to also be addressed. These factors may lead to altered and unreliable BOLD effects as seen on the conventional task-based fMRI, such as neurovascular uncoupling and head motion (for a thorough review of these issues, see Silva et al. ${ }^{58}$ ).

\section{Future Directions}

As Briggs et al. mentioned, ${ }^{6}$ we may be astounded when we look back on these days of relatively uninformed surgical decision-making-when we estimated operative risks and planned approaches without knowing the functional connectivity of an individual patient's brain. This is often the case when we hear stories of tumor localization based on cerebral angiography, early frontal lobotomies, and exploratory trephining of the skull. New technology can now be applied to old methods to expand our understanding of brain function. For example, using lesion network mapping in patients with common symptoms but various lesion locations, disrupted networks can be retrospectively identified and new treatment targets suggested. ${ }^{18}$ As neurosurgeons perform safer and more precise operations, the expectation of functional optimization expands the definition of "eloquent" brain. ${ }^{21}$ Ultimately, adoption of rs-fMRI for the diagnosis and management of neurosurgical diseases will require better anatomical visualization of RSN outputs that do not simply overlay colored maps on standardized digital brain models without the elegant 
anatomical detail that neurosurgeons are accustomed to in the operating room. For example, Fig. 2 shows four of the networks discussed above mapped onto a single patient's native T1-weighted postcontrast MRI (the same patient as in Fig. 1). In this regard, those who have successfully integrated rs-fMRI into clinical practice have already set the standards upon which the field can improve..$^{4,35,41}$

\section{Conclusions}

Rs-fMRI for cognitive and emotional network mapping in neurosurgical patients is feasible and has the potential to identify critical brain networks that are not traditionally "eloquent." Correlating rs-fMRI results with gold-standard DES and neuropsychological outcomes is imperative in order to support its universal adoption into neurosurgical management. At present, rs-fMRI is a promising adjunct to DES but is too unreliable for use alone during lesion resection. However, it is possible that continued comprehensive functional mapping of brain networks in patients with epilepsy and brain tumors may provide the momentum for rs-fMRI to penetrate deep into the clinical realm and to realize its true potential in other neurological diseases.

\section{References}

1. Bar M, Aminoff E: Cortical analysis of visual context. Neuron 38:347-358, 2003

2. Berger MS, Ojemann GA, Lettich E: Neurophysiological monitoring during astrocytoma surgery. Neurosurg Clin $\mathbf{N}$ Am 1:65-80, 1990

3. Biswal B, Yetkin FZ, Haughton VM, Hyde JS: Functional connectivity in the motor cortex of resting human brain using echo-planar MRI. Magn Reson Med 34:537-541, 1995

4. Boerwinkle VL, Mohanty D, Foldes ST, Guffey D, Minard CG, Vedantam A, et al: Correlating resting-state functional magnetic resonance imaging connectivity by independent component analysis-based epileptogenic zones with intracranial electroencephalogram localized seizure onset zones and surgical outcomes in prospective pediatric intractable epilepsy study. Brain Connect 7:424-442, 2017

5. Böttger J, Margulies DS, Horn P, Thomale UW, Podlipsky I, Shapira-Lichter I, et al: A software tool for interactive exploration of intrinsic functional connectivity opens new perspectives for brain surgery. Acta Neurochir (Wien) 153:1561-1572, 2011

6. Briggs RG, Conner AK, Baker CM, Burks JD, Glenn CA, Sali G, et al: A connectomic atlas of the human cerebrumChapter 18: The connectional anatomy of human brain networks. Oper Neurosurg (Hagerstown) 15 (suppl_1):S470S480, 2018

7. Buckner RL, DiNicola LM: The brain's default network: updated anatomy, physiology and evolving insights. Nat Rev Neurosci 20:593-608, 2019

8. Bugalho P, Alves L, Miguel R, Ribeiro O: Profile of cognitive dysfunction and relation with gait disturbance in normal pressure hydrocephalus. Clin Neurol Neurosurg 118:83-88, 2014

9. Cole DM, Smith SM, Beckmann CF: Advances and pitfalls in the analysis and interpretation of resting-state FMRI data. Front Syst Neurosci 4:8, 2010

10. Cole MW, Reynolds JR, Power JD, Repovs G, Anticevic A, Braver TS: Multi-task connectivity reveals flexible hubs for adaptive task control. Nat Neurosci 16:1348-1355, 2013

11. Dixon ML, De La Vega A, Mills C, Andrews-Hanna J, Spreng RN, Cole MW, et al: Heterogeneity within the fron- toparietal control network and its relationship to the default and dorsal attention networks. Proc Natl Acad Sci U S A 115:E1598-E1607, 2018

12. Duchek JM, Balota DA, Thomas JB, Snyder AZ, Rich P, Benzinger TL, et al: Relationship between Stroop performance and resting state functional connectivity in cognitively normal older adults. Neuropsychology 27:516-528, 2013

13. Duffau H: The error of Broca: from the traditional localizationist concept to a connectomal anatomy of human brain. $\mathbf{J}$ Chem Neuroanat 89:73-81, 2018

14. Duffau H, Capelle L, Sichez N, Denvil D, Lopes M, Sichez JP, et al: Intraoperative mapping of the subcortical language pathways using direct stimulations. An anatomo-functional study. Brain 125:199-214, 2002

15. Duncan J: The multiple-demand (MD) system of the primate brain: mental programs for intelligent behaviour. Trends Cogn Sci 14:172-179, 2010

16. Eickhoff SB, Thirion B, Varoquaux G, Bzdok D: Connectivity-based parcellation: Critique and implications. Hum Brain Mapp 36:4771-4792, 2015

17. Fornito A, Zalesky A, Bullmore ET (eds): Fundamentals of Brain Network Analysis. London: Elsevier, 2016

18. Fox MD: Mapping symptoms to brain networks with the human connectome. N Engl J Med 379:2237-2245, 2018

19. Fox MD, Raichle ME: Spontaneous fluctuations in brain activity observed with functional magnetic resonance imaging. Nat Rev Neurosci 8:700-711, 2007

20. Fox MD, Snyder AZ, Vincent JL, Corbetta M, Van Essen DC, Raichle ME: The human brain is intrinsically organized into dynamic, anticorrelated functional networks. Proc Natl Acad Sci U S A 102:9673-9678, 2005

21. Fried I: The myth of eloquent cortex, or what is non-eloquent cortex? J Neurosurg 78:1009-1010, 1993

22. Gilmore AW, Nelson SM, McDermott KB: A parietal memory network revealed by multiple MRI methods. Trends Cogn Sci 19:534-543, 2015

23. Gray JD, Milner TA, McEwen BS: Dynamic plasticity: the role of glucocorticoids, brain-derived neurotrophic factor and other trophic factors. Neuroscience 239:214-227, 2013

24. Griffanti L, Douaud G, Bijsterbosch J, Evangelisti S, AlfaroAlmagro F, Glasser MF, et al: Hand classification of fMRI ICA noise components. Neuroimage 154:188-205, 2017

25. Hart MG, Romero-Garcia R, Price SJ, Suckling J: Global effects of focal brain tumors on functional complexity and network robustness: a prospective cohort study. Neurosurgery 84:1201-1213, 2019

26. Hellström P, Edsbagge M, Archer T, Tisell M, Tullberg M, Wikkelsø C: The neuropsychology of patients with clinically diagnosed idiopathic normal pressure hydrocephalus. Neurosurgery 61:1219-1228, 2007

27. Herbet G, Maheu M, Costi E, Lafargue G, Duffau H: Mapping neuroplastic potential in brain-damaged patients. Brain 139:829-844, 2016

28. Herbet G, Moritz-Gasser S: Beyond language: mapping cognition and emotion. Neurosurg Clin N Am 30:75-83, 2019

29. Jiang H, He NY, Sun YH, Jian FF, Bian LG, Shen JK, et al: Altered spontaneous brain activity in Cushing's disease: a resting-state functional MRI study. Clin Endocrinol (Oxf) 86:367-376, 2017

30. Kaiser RH, Andrews-Hanna JR, Wager TD, Pizzagalli DA: Large-scale network dysfunction in major depressive disorder: a meta-analysis of resting-state functional connectivity. JAMA Psychiatry 72:603-611, 2015

31. Khoo HM, Kishima H, Tani N, Oshino S, Maruo T, Hosomi $\mathrm{K}$, et al: Default mode network connectivity in patients with idiopathic normal pressure hydrocephalus. J Neurosurg 124:350-358, 2016

32. Kwong KK, Belliveau JW, Chesler DA, Goldberg IE, Weisskoff RM, Poncelet BP, et al: Dynamic magnetic resonance 
imaging of human brain activity during primary sensory stimulation. Proc Natl Acad Sci U S A 89:5675-5679, 1992

33. Lee MH, Smyser CD, Shimony JS: Resting-state fMRI: a review of methods and clinical applications. AJNR Am J Neuroradiol 34:1866-1872, 2013

34. Leuthardt EC, Allen M, Kamran M, Hawasli AH, Snyder AZ, Hacker CD, et al: Resting-state blood oxygen level-dependent functional MRI: a paradigm shift in preoperative brain mapping. Stereotact Funct Neurosurg 93:427-439, 2015

35. Leuthardt EC, Guzman G, Bandt SK, Hacker C, Vellimana AK, Limbrick D, et al: Integration of resting state functional MRI into clinical practice-a large single institution experience. PLoS One 13: 0198349,2018

36. Lu J, Zhang H, Hameed NUF, Zhang J, Yuan S, Qiu T, et al: An automated method for identifying an independent component analysis-based language-related resting-state network in brain tumor subjects for surgical planning. Sci Rep 7:13769, 2017

37. Marek S, Dosenbach NUF: The frontoparietal network: function, electrophysiology, and importance of individual precision mapping. Dialogues Clin Neurosci 20:133-140, 2018

38. McKeown MJ, Makeig S, Brown GG, Jung TP, Kindermann SS, Bell AJ, et al: Analysis of fMRI data by blind separation into independent spatial components. Hum Brain Mapp 6:160-188, 1998

39. Menon V: Large-scale brain networks and psychopathology: a unifying triple network model. Trends Cogn Sci 15:483506, 2011

40. Menon V: Salience network, in Toga AW (ed): Brain Mapping: An Encyclopedic Reference. London: Elsevier, 2015, Vol 2, pp 597-611

41. Mitchell TJ, Hacker CD, Breshears JD, Szrama NP, Sharma M, Bundy DT, et al: A novel data-driven approach to preoperative mapping of functional cortex using resting-state functional magnetic resonance imaging. Neurosurgery 73:969-983, 2013

42. Oathes DJ, Patenaude B, Schatzberg AF, Etkin A: Neurobiological signatures of anxiety and depression in resting-state functional magnetic resonance imaging. Biol Psychiatry 77:385-393, 2015

43. Ojemann GA: Individual variability in cortical localization of language. J Neurosurg 50:164-169, 1979

44. Orringer DA, Vago DR, Golby AJ: Clinical applications and future directions of functional MRI. Semin Neurol 32:466475, 2012

45. Papez JW: A proposed mechanism of emotion. 1937. J Neuropsychiatry Clin Neurosci 7:103-112, 1995

46. Penfield W, Boldrey E: Somatic motor and sensory representation in the cerebral cortex of man as studied by electrical stimulation. Brain 60:389-443, 1937

47. Pessoa L: A network model of the emotional brain. Trends Cogn Sci 21:357-371, 2017

48. Purves D, Augustine GJ, Fitzpatrick D, Katz LC, LaMantia AS, McNamara JO, et al (eds): The limbic system, in Neuroscience, ed 2. Sunderland, MA: Sinauer Associates, 2001

49. Raichle ME: The brain's default mode network. Annu Rev Neurosci 38:433-447, 2015

50. Raichle ME: Neuroscience. The brain's dark energy. Science 314:1249-1250, 2006

51. Roland JL, Griffin N, Hacker CD, Vellimana AK, Akbari SH, Shimony JS, et al: Resting-state functional magnetic resonance imaging for surgical planning in pediatric patients: a preliminary experience. J Neurosurg Pediatr 20:583-590, 2017

52. Russell G, Lightman S: The human stress response. Nat Rev Endocrinol 15:525-534, 2019

53. Sair HI, Yahyavi-Firouz-Abadi N, Calhoun VD, Airan RD, Agarwal S, Intrapiromkul J, et al: Presurgical brain mapping of the language network in patients with brain tumors using resting-state fMRI: comparison with task fMRI. Hum Brain Mapp 37:913-923, 2016

54. Sarubbo S, Tate M, De Benedictis A, Merler S, MoritzGasser S, Herbet G, et al: Mapping critical cortical hubs and white matter pathways by direct electrical stimulation: an original functional atlas of the human brain. Neuroimage 205:116237, 2019

55. Seitzman BA, Snyder AZ, Leuthardt EC, Shimony JS: The state of resting state networks. Top Magn Reson Imaging 28:189-196, 2019

56. Shimony JS, Zhang D, Johnston JM, Fox MD, Roy A, Leuthardt EC: Resting-state spontaneous fluctuations in brain activity: a new paradigm for presurgical planning using fMRI. Acad Radiol 16:578-583, 2009

57. Shulman GL, Fiez JA, Corbetta M, Buckner RL, Miezin FM, Raichle ME, et al: Common blood flow changes across visual tasks: II. Decreases in cerebral cortex. J Cogn Neurosci 9:648-663, 1997

58. Silva MA, See AP, Essayed WI, Golby AJ, Tie Y: Challenges and techniques for presurgical brain mapping with functional MRI. Neuroimage Clin 17:794-803, 2017

59. Simmons NE, Do HM, Lipper MH, Laws ER Jr: Cerebral atrophy in Cushing's disease. Surg Neurol 53:72-76, 2000

60. Skrap M, Marin D, Ius T, Fabbro F, Tomasino B: Brain mapping: a novel intraoperative neuropsychological approach. J Neurosurg 125:877-887, 2016

61. Smith SM, Miller KL, Salimi-Khorshidi G, Webster M, Beckmann CF, Nichols TE, et al: Network modelling methods for FMRI. Neuroimage 54:875-891, 2011

62. Squire LR, Zola-Morgan S: The medial temporal lobe memory system. Science 253:1380-1386, 1991

63. Suri S, Topiwala A, Filippini N, Zsoldos E, Mahmood A, Sexton CE, et al: Distinct resting-state functional connections associated with episodic and visuospatial memory in older adults. Neuroimage 159:122-130, 2017

64. Tie Y, Rigolo L, Norton IH, Huang RY, Wu W, Orringer D, et al: Defining language networks from resting-state fMRI for surgical planning - a feasibility study. Hum Brain Mapp 35:1018-1030, 2014

65. Tie Y, Whalen S, Suarez RO, Golby AJ: Group independent component analysis of language fMRI from word generation tasks. Neuroimage 42:1214-1225, 2008

66. van den Heuvel MP, Mandl RCW, Kahn RS, Hulshoff Pol HE: Functionally linked resting-state networks reflect the underlying structural connectivity architecture of the human brain. Hum Brain Mapp 30:3127-3141, 2009

67. Vincent JL, Snyder AZ, Fox MD, Shannon BJ, Andrews JR, Raichle ME, et al: Coherent spontaneous activity identifies a hippocampal-parietal memory network. J Neurophysiol 96:3517-3531, 2006

68. Vossel S, Geng JJ, Fink GR: Dorsal and ventral attention systems: distinct neural circuits but collaborative roles. Neuroscientist 20:150-159, 2014

69. Williams MA, Malm J: Diagnosis and treatment of idiopathic normal pressure hydrocephalus. Continuum (Minneap Minn) 22 (2 Dementia):579-599, 2016

70. Yordanova YN, Cochereau J, Duffau H, Herbet G: Combining resting state functional MRI with intraoperative cortical stimulation to map the mentalizing network. Neuroimage 186:628-636, 2019

71. Zacà D, Jovicich J, Corsini F, Rozzanigo U, Chioffi F, Sarubbo S: ReStNeuMap: a tool for automatic extraction of restingstate functional MRI networks in neurosurgical practice. J Neurosurg 131:764-771, 2018

72. Zang Y, Jiang T, Lu Y, He Y, Tian L: Regional homogeneity approach to fMRI data analysis. Neuroimage 22:394-400, 2004

73. Zang YF, He Y, Zhu CZ, Cao QJ, Sui MQ, Liang M, et al: Altered baseline brain activity in children with ADHD 
revealed by resting-state functional MRI. Brain Dev 29:8391, 2007

74. Zhang D, Johnston JM, Fox MD, Leuthardt EC, Grubb RL, Chicoine MR, et al: Preoperative sensorimotor mapping in brain tumor patients using spontaneous fluctuations in neuronal activity imaged with functional magnetic resonance imaging: initial experience. Neurosurgery 65 (6 Suppl):226-236, 2009

75. Zhang Z, Lu G, Zhong Y, Tan Q, Yang Z, Liao W, et al: Impaired attention network in temporal lobe epilepsy: a resting FMRI study. Neurosci Lett 458:97-101, 2009

\section{Disclosures}

The authors report no conflict of interest concerning the materials or methods used in this study or the findings specified in this paper.

\section{Author Contributions}

Conception and design: Tie, Catalino, Golby. Acquisition of data: Tie, Yao. Analysis and interpretation of data: Tie, Catalino, Yao. Drafting the article: Tie, Catalino, Yao, Green, Laws. Critically revising the article: all authors. Reviewed submitted version of manuscript: Tie, Catalino, Yao, Laws, Golby. Approved the final version of the manuscript on behalf of all authors: Tie. Statistical analysis: Yao. Administrative/technical/material support: Tie, Yao. Study supervision: Tie.

\section{Correspondence}

Yanmei Tie: Brigham and Women's Hospital, Harvard Medical School, Boston, MA. ytie@bwh.harvard.edu. 